\title{
An Effect of Color Channel Replacement
}

\author{
Gwanggil Jeon \\ Department of Embedded Systems Engineering, Incheon National University \\ 119 Academy-ro, Yeonsu-gu, Incheon 406-772, Korea
}

gjeon@incheon.ac.kr

\begin{abstract}
This paper investigates the effect of color channel replacement with histogram equalization. The histogram equalization is broadly employed for contrast intensification in a diverse of consumer electronics applications for its stratghorward function. The proposed work is consists of seven steps, original given image, channek sepandtion: color image to three gray scale image, color channel replacemen, HEM on individually separated image, histogram modification in each color channel, reconstruct color images, and image display. Simulation results with test images are provided to compåre its PSNR and MSE performance.
\end{abstract}

Keywords: Image, histogram equalization, color indoe, color replacement

\section{Introduction}

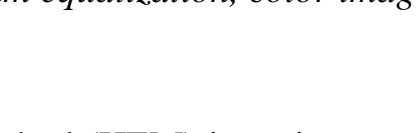

Histogram equalization method (HEM) is an important field in image processing for both human and computer vision. HEM changes original images in different ways, and this application includes photographs, photochemical photographs, and illustrations. The higher quality of images enables operators to manage state-of-art approaches for image enhancement [1-3]. HEM adjusts pixel intensit)es and the histogram of the resulting image becomes uniform.

HEM is broadly adopted in many applications including speech recognition, texture synthesis, medical image processing, and many other video processing applications [4-8]. There have been many applications to develop HEM [9]. Most of them employed straightforward linear or nonlinear gray level transformation functions. Some others have been using more complex functions or features such as edge or colors [10-12]. By all means, HEM is the most usually adopted approach due to its plainness and comparatively better performanof on almost all kinds of images.

Ceneranly speaking, HEM levels the density distribution of the derived images and increases the contrast of the image as a consequence. It is because HEM has an influence of dynamic range extending. However, despite of its better performance in intensifying contrasts of a provided image, it is hardly employed in consumer electronics applications such as television due to its straightforward usage of HEM, and its effect may affect the original brightness of an input image and eventually spoil the subjective quality.

In this paper, we used global HEM. The global HEM uses the histogram information of the whole input images for its transformation function. We assume gray scale image and color image are 1-D and 3-D approach as color image has three color channels [13]. The rest of this paper is composed as follows. In Section 2, we explain the proposed method. Section 3 shows simulation results and conclusion remarks are shown in Section 4. 


\section{Proposed method}

Histogram equation method (HEM) intensifies the images contrast by changing the intensity values of given images. Sometimes it can be used for indexed colormap image values. The HEM normally enhances the universal contrast of images. It is useful when the adopted image data is outlined with close contrast intensities. In this case, the strengths can be adjusted by changing the intensities of the histogram. This is the main idea that one may enhance low contrast image to high contrast image. One of the main benefits of HEM is that HEM is a simple approach. Therefore, if one knows the histogram equalization function, then the original low contrast image can be regained perfectly. Let us assume a discontinuous grayscale image $x$, and suppose $k_{i}$ be the number of events of gray level $i$. The probability of an event of a pixel of level $i$ in the original image is

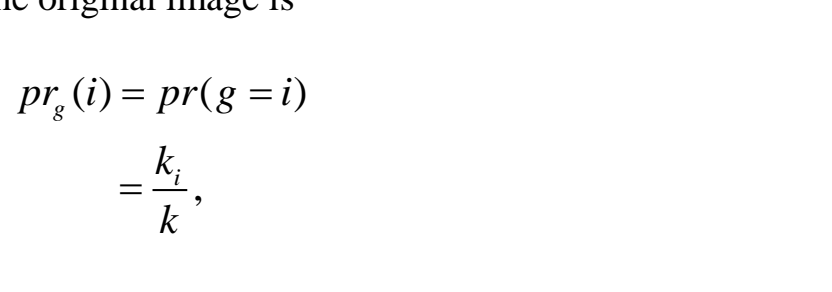

where $0<i<L$. We assume $L$ is the total number of gray levelsin the image. Since we assumed $g$ as grey image, $L$ is 256 . Parameter $k$ is the number of pixels in an image. Then, the cumulative distribution function is,

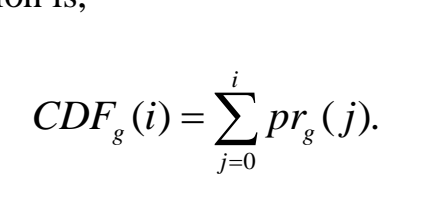

Here, CDF is cumulative distribution function which depicts the probability that a realvalued random variable $M$ with a granted probability distribution is discovered at a value equal to or less than $m$. Now, to map the values back into their original range, the following simple transformation needs to be applied on the result:

$$
h^{\prime}=h \cdot\left(g_{\max }-g_{\text {min }}\right)+g_{\text {min }} \text {. }
$$

This is the global histogram equalization and we use this approach in the proposed method.

Although above HEM is for the grayscale image, it can also be employed on color images by applying the HEM individually to red, green and blue channels of color image. The study compares the effect of HEM on color replaced images. Figure 1 shows the block diagram of the proposed method. The proposed method has seven steps.

Step 1: Original given image

Step 2: Channel separation: Color image to three gray scale image

Step 3: Color channel replacement

Step 4: HEM on individually separated image

Step 5: Histogram modification in each color channel

Step 6: Reconstruct color images

Step 7: Image display 

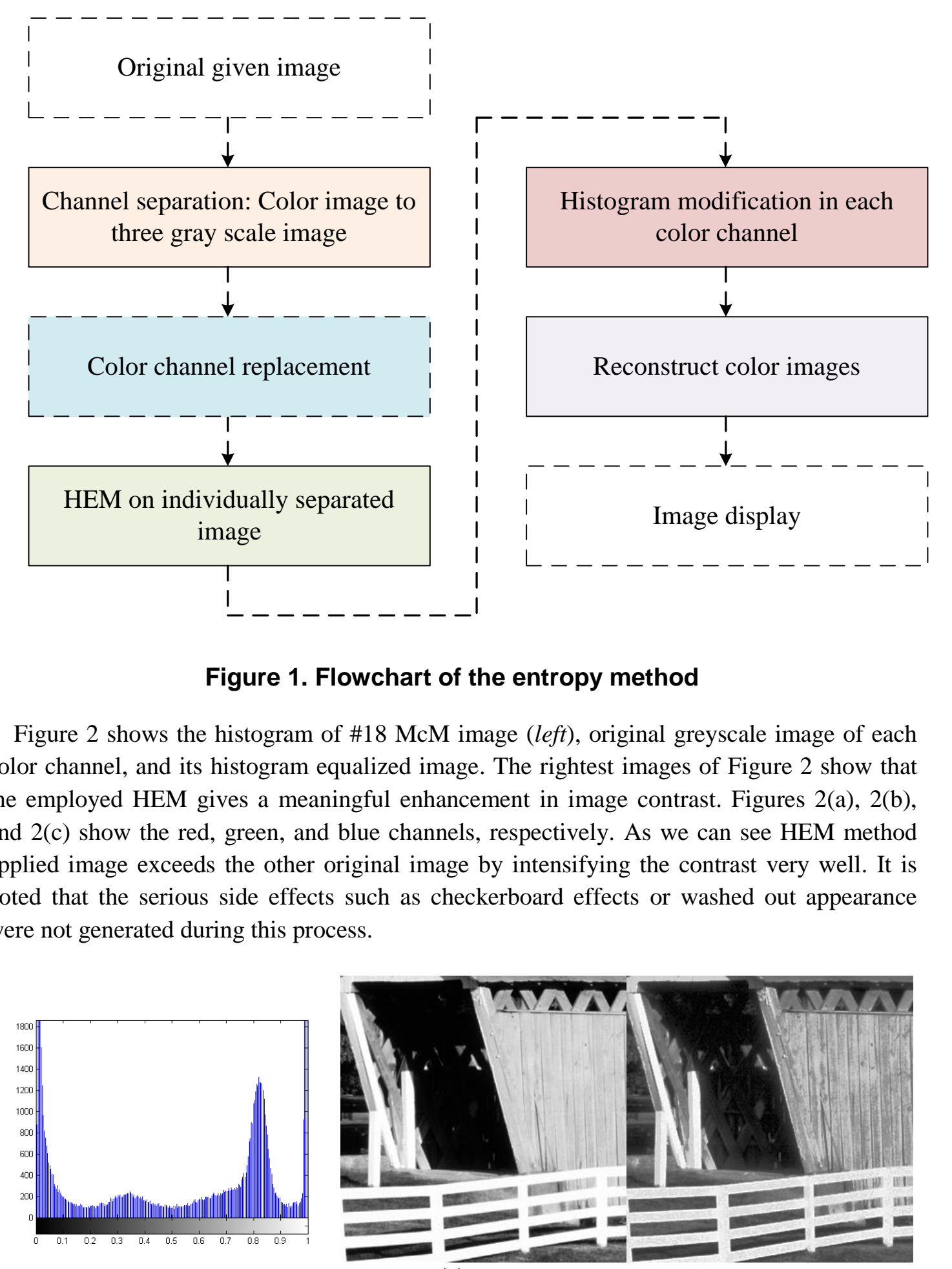

(a) 

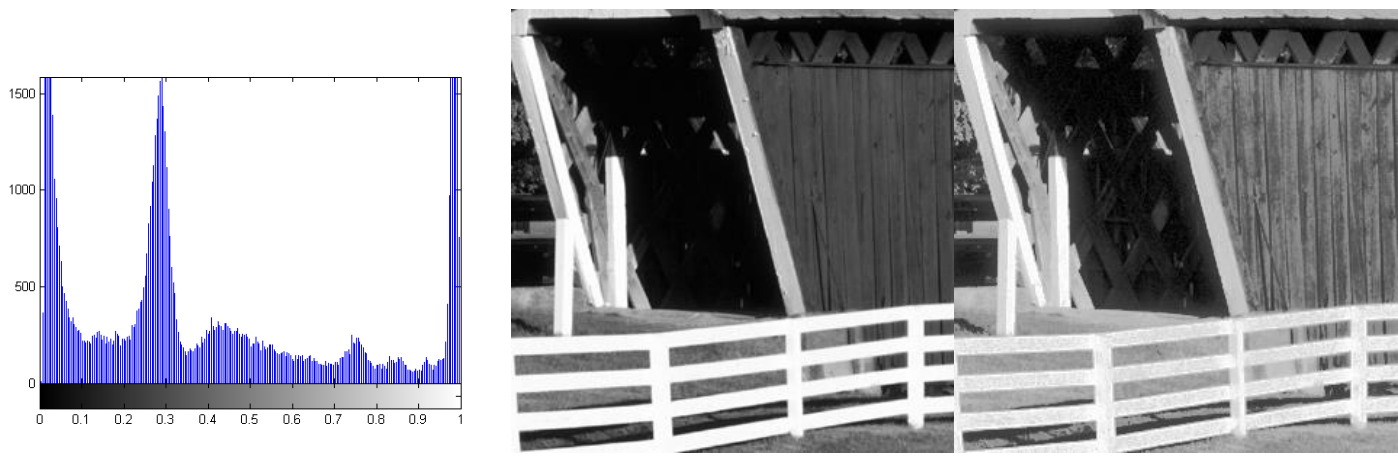

(b)
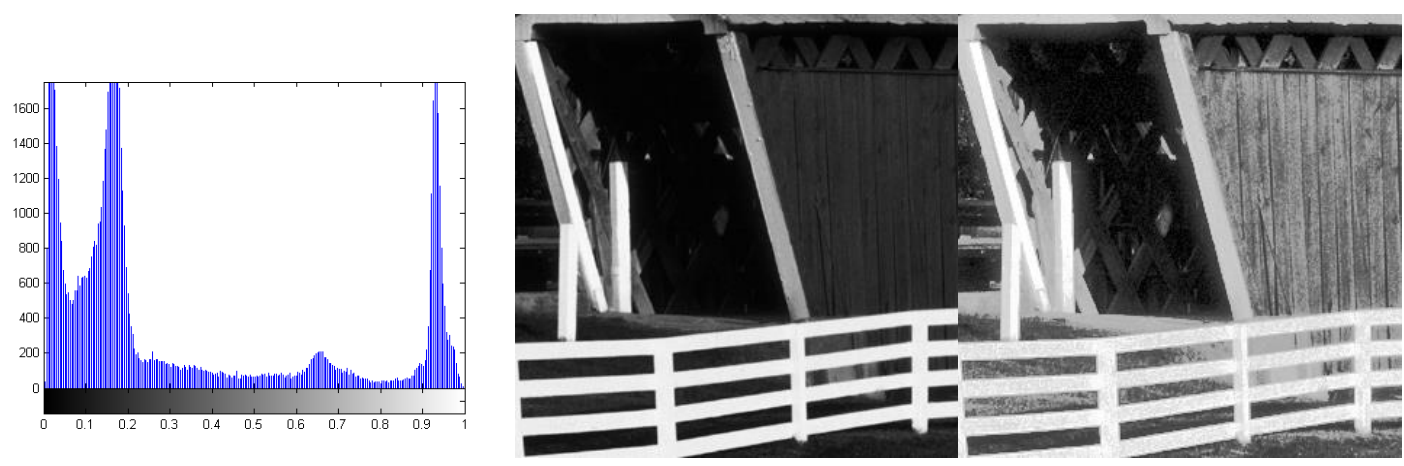

(c)

Figure 2. Histogram of \#18 McM image and its original color channel and its histogram equalized image: (a) red (b) green, and (c) blue. Note that the leftist image shows the original histogram and the rightest image is with specified

\section{Simulation results}

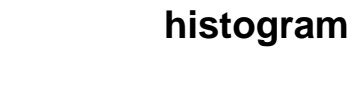

The simulation results from introduced method were conducted on several images and assessed the performance. The simulation was conducted on $18 \mathrm{McM}$ test images, which are shown in Figure 3.

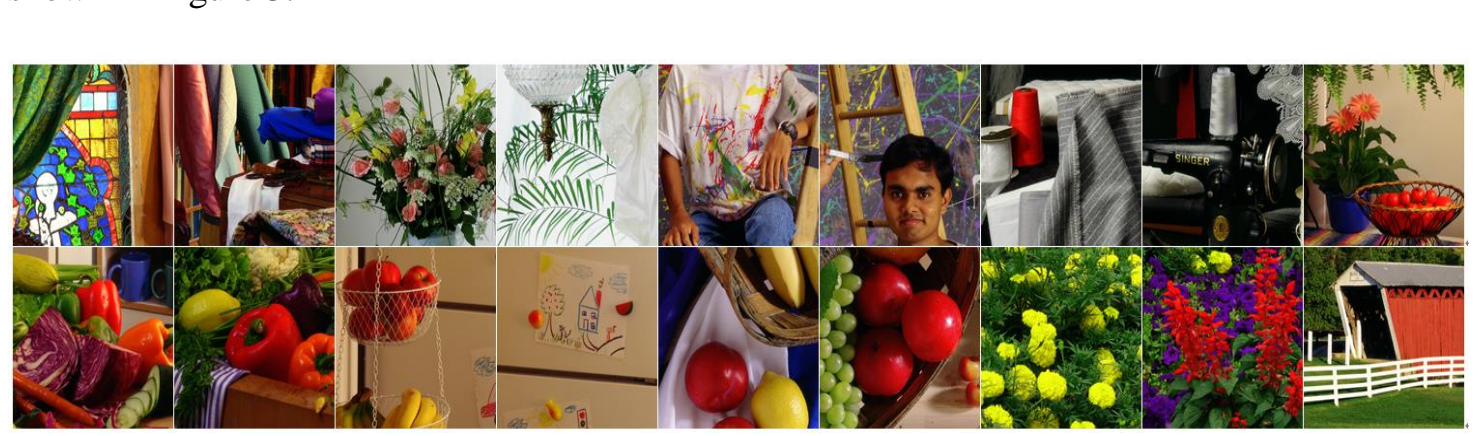

Figure 3. Eighteen test image set: McM images \#1 to \#18 


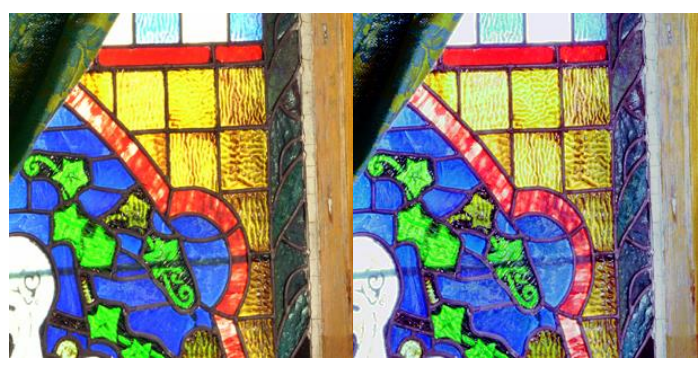

(a)
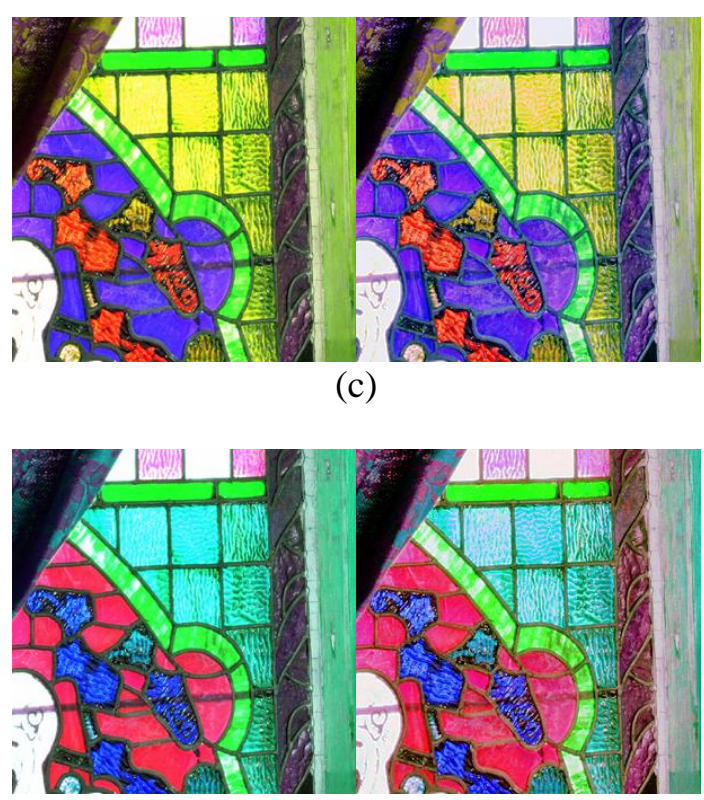

(e) (c)

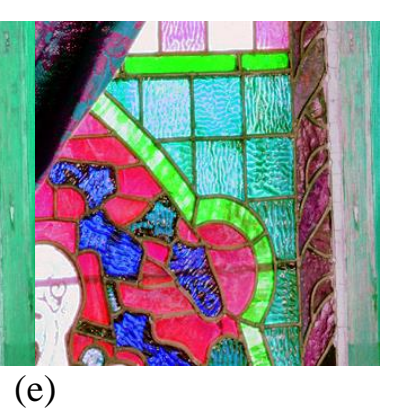

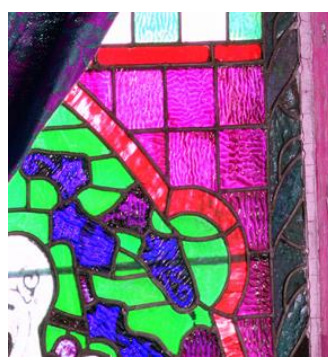

(b)

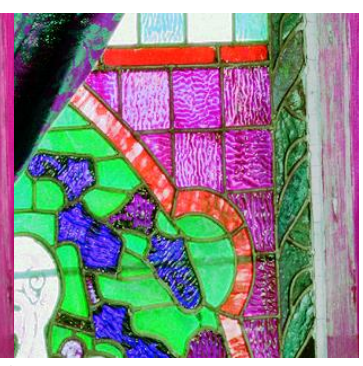

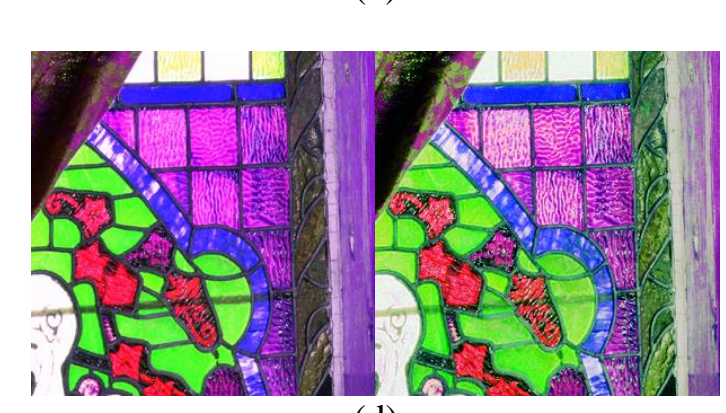

(d)
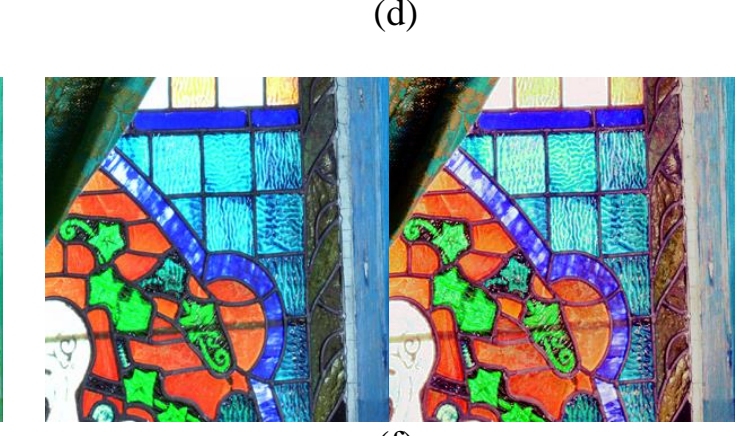

(f)

Figure 4. Result images of McM \#1 image with different combination of R, G, B channels and its histogram equalized results: (a) RGB, (b) RBG, (c) GRB, (d) GBR, (e) BRG, and (f) BGR

Figure 4(a) shows the RGB original image along with simulation results from HEM on McM image 11 In the same manner, Figures 4(b-f) shows different combination of red, green and blue color channels along with simulation results from HEM. They are RBG, GRB, GBR, BRG, and BGR, respectively.

In the same manner, we obtained two other examples with McM images \#5 and \#14, which are shown in Figures 5-6.

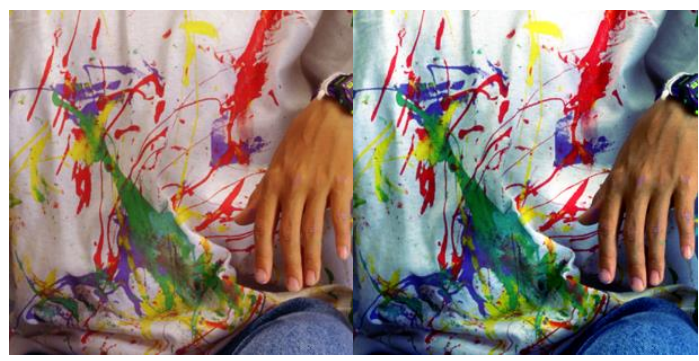

(a)

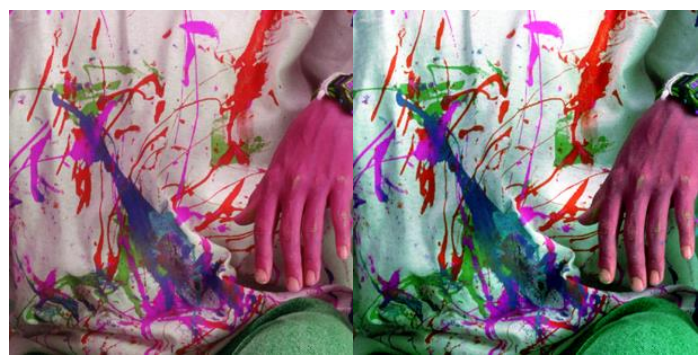

(b) 


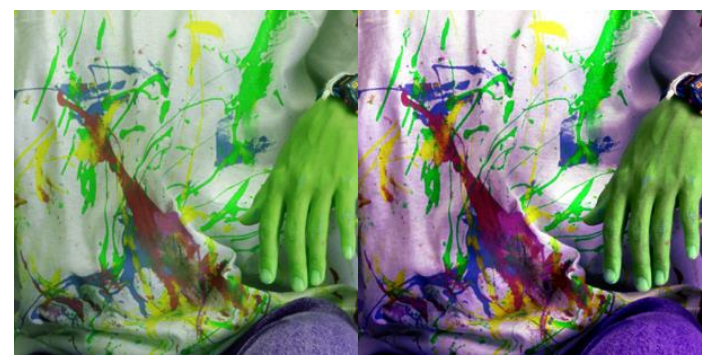

(c)

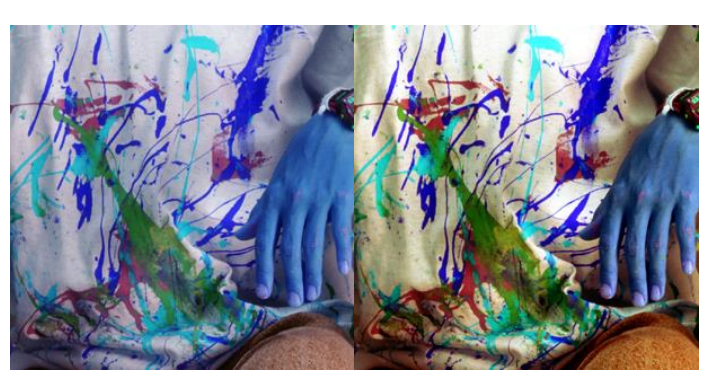

(e)

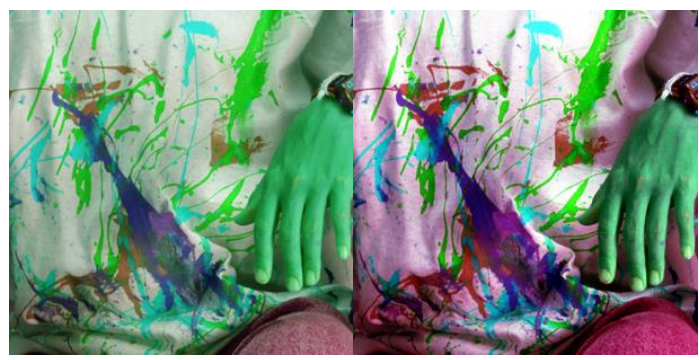

(d)

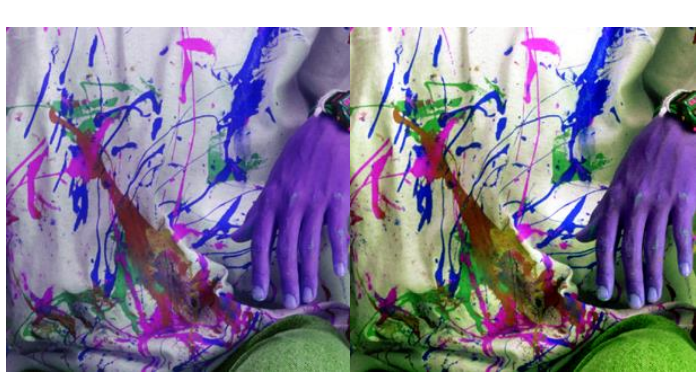

(f)

Figure 5. Result images of McM \#5 image with different combination of R, G, B channels and its histogram equalized results? (a) RGB, (b) RBG, (c) GRB, (d) GBR, (e) BRG, and (f) BGR

Table 1 shows the PSNR comparison for $18 \mathrm{McM}$ images (\#1 to \#18 images) with replaced red, green, and blue combinations. Figures 7 and 8 show the performance comparisons in CPSNR $(\mathrm{dB})$ and CMSE for replaced red, green, and blue combinations.

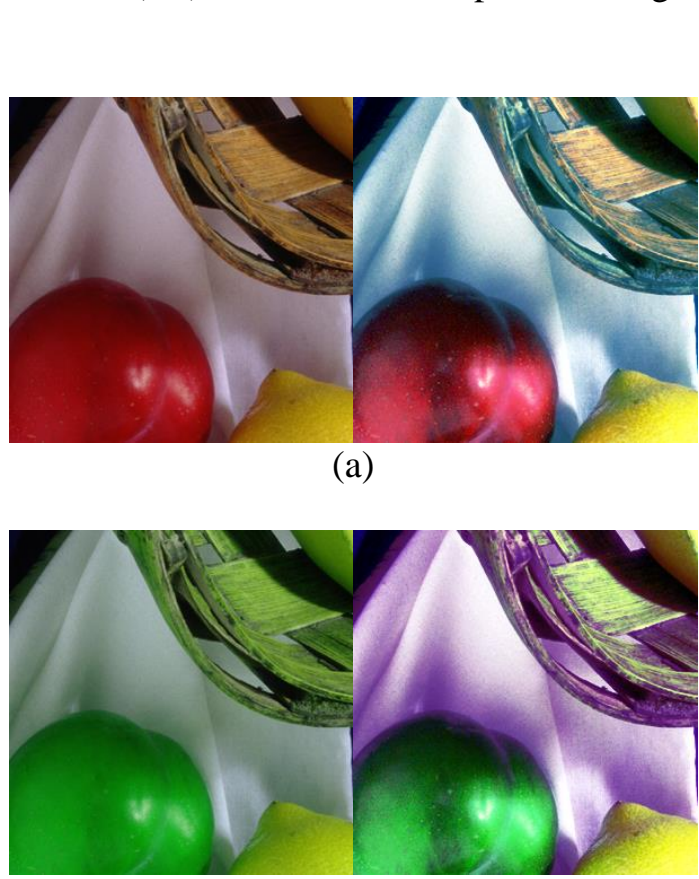

(c)

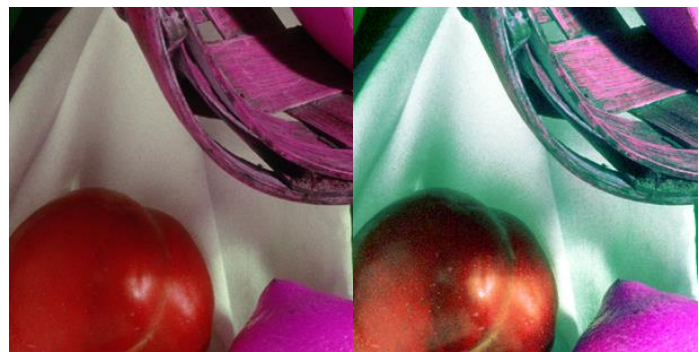

(b)

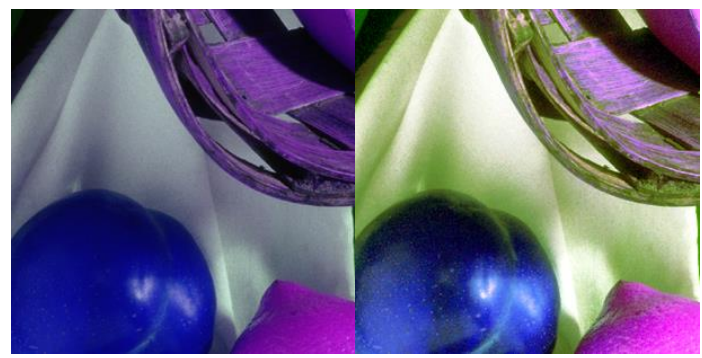

(d) 


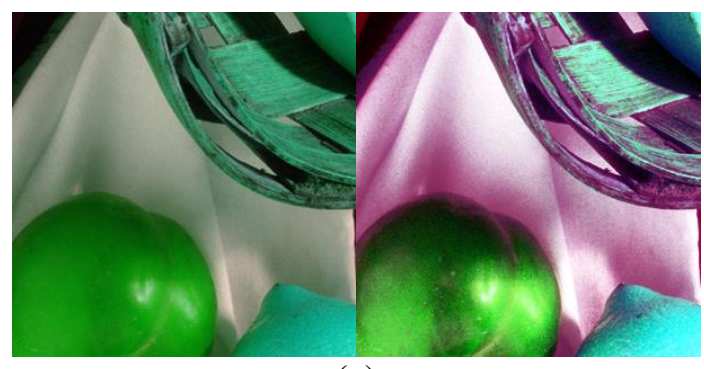

(e)

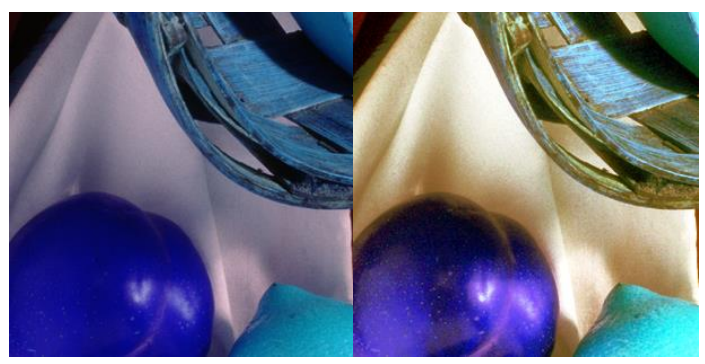

(f)

Figure 6. Result images of McM \#14 image with different combination of $R, Q, B$ channels and its histogram equalized results: (a) RGB, (b) RBG, (c) GRB, (d) GBR, (e) BRG, and (f) BGR

Table 1. PSNR comparison for $18 \mathrm{McM}$ images ( $1^{\text {st }}$ to $18^{\mathrm{h}}$ images) with varying red, green, and blue combinations

\begin{tabular}{|c|c|c|c|c|c|c|c|c|c|}
\hline & \multicolumn{3}{|c|}{ R channel } & \multicolumn{3}{|c|}{ G channel } & \multicolumn{3}{|c|}{ B channel } \\
\hline & RGB & GBR & BRG & RGB & GBR & BRG & RGB & GBR & BRG \\
\hline 1 & 23.604 & 11.053 & 7.449 & & 8.944 & 14425 & 14.846 & 6.793 & 8.150 \\
\hline 2 & 17.372 & 14.247 & 11.243 & & & 13.616 & 16.409 & 10.975 & 12.995 \\
\hline 3 & 17.515 & 14.827 & 15.514 & & 15.510 & 14.545 & 11.750 & 11.145 & 11.425 \\
\hline 4 & 13.125 & 13.029 & 13.082 & 11.171 & 11.078 & 11.018 & 13.401 & 13.213 & 13.222 \\
\hline 5 & 16.410 & 12.583 & (1) 286 & & 15.207 & 13.914 & 20.710 & 11.898 & 14.819 \\
\hline 6 & 16.973 & & 10.885 & & 10.564 & 11.506 & 10.312 & 8.783 & 9.575 \\
\hline 7 & 20.105 & & 12.088 & 15.282 & 15.204 & 11.380 & 14.434 & 11.050 & 14.427 \\
\hline 8 & & & & 8.329 & 8.278 & 8.225 & 7.818 & 7.739 & 7.810 \\
\hline 9 & 20.526 & 11.975 & $(12.254$ & 16.011 & 14.729 & 11.754 & 11.062 & 9.405 & 10.598 \\
\hline 10 & 21.850 & 10.374 & 9.446 & 8.837 & 7.523 & 7.208 & 7.827 & 6.654 & 7.221 \\
\hline 11 & 18.814 & $8-662$ & 8.387 & 8.528 & 6.914 & 6.378 & 6.203 & 5.272 & 6.059 \\
\hline 12 & 13.992 & 12.442 & 12.081 & 13.142 & 12.792 & 11.496 & 9.224 & 8.552 & 9.121 \\
\hline 13 & 12.446 & 11.906 & 11.557 & 13.698 & 13.392 & 12.577 & 11.385 & 10.471 & 11.165 \\
\hline 14 & & 13.023 & 12.407 & 13.216 & 11.863 & 10.598 & 12.085 & 9.623 & 11.474 \\
\hline 13 & 21.135 & 13.353 & 12.807 & 6.522 & 6.340 & 5.670 & 6.265 & 5.639 & 6.382 \\
\hline 16 & 14.379 & 14.090 & 7.076 & 20.607 & 8.535 & 19.404 & 6.003 & 5.651 & 5.681 \\
\hline 17 & 18.022 & 7.444 & 7.278 & 8.053 & 6.594 & 5.963 & 8.412 & 6.233 & 6.091 \\
\hline 18 & 17.844 & 13.162 & 14.692 & 17.687 & 16.427 & 13.425 & 12.281 & 11.160 & 12.314 \\
\hline Avg. & 17.166 & 12.069 & 10.982 & 13.911 & 11.232 & 11.117 & 11.135 & 8.903 & 9.918 \\
\hline
\end{tabular}


International Journal of Multimedia and Ubiquitous Engineering

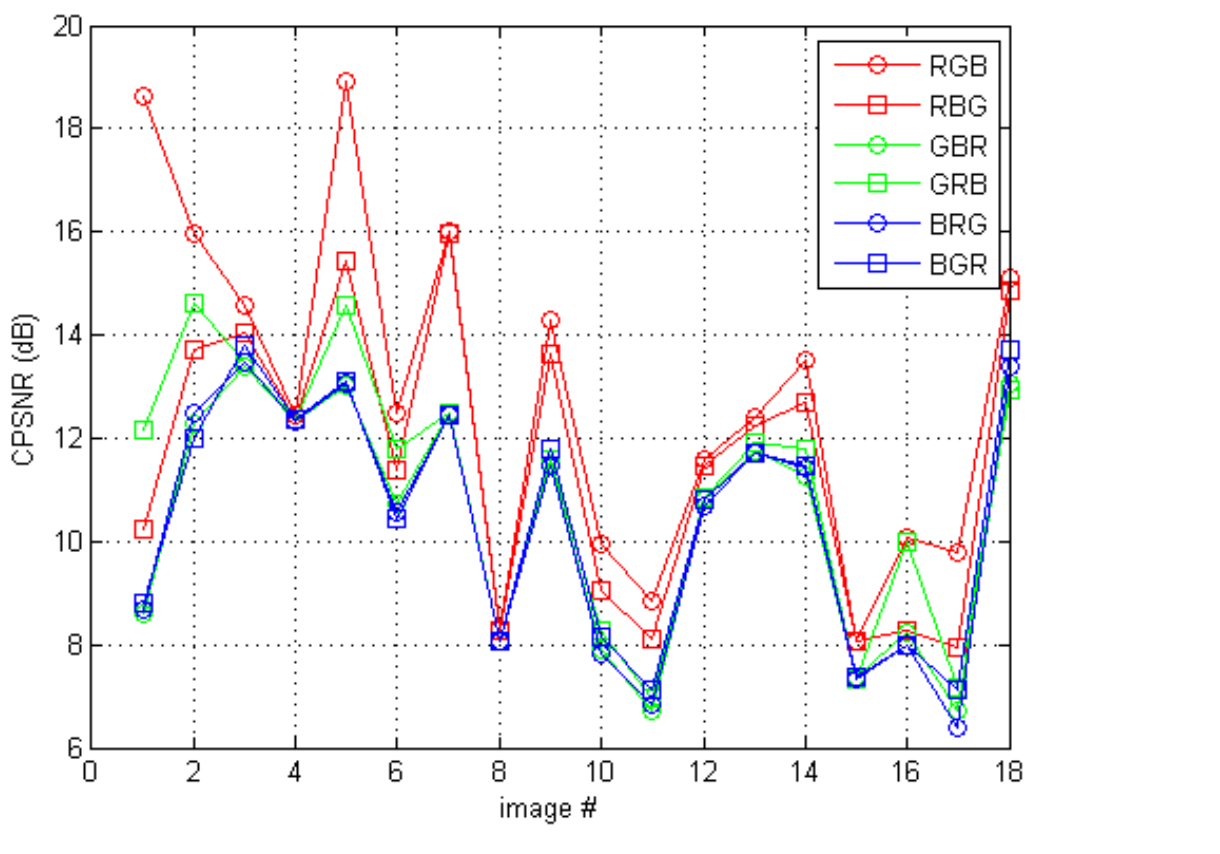

Figure 7. Performance comparison in CPSNR (dB): different combination of red, green, and blue combinations

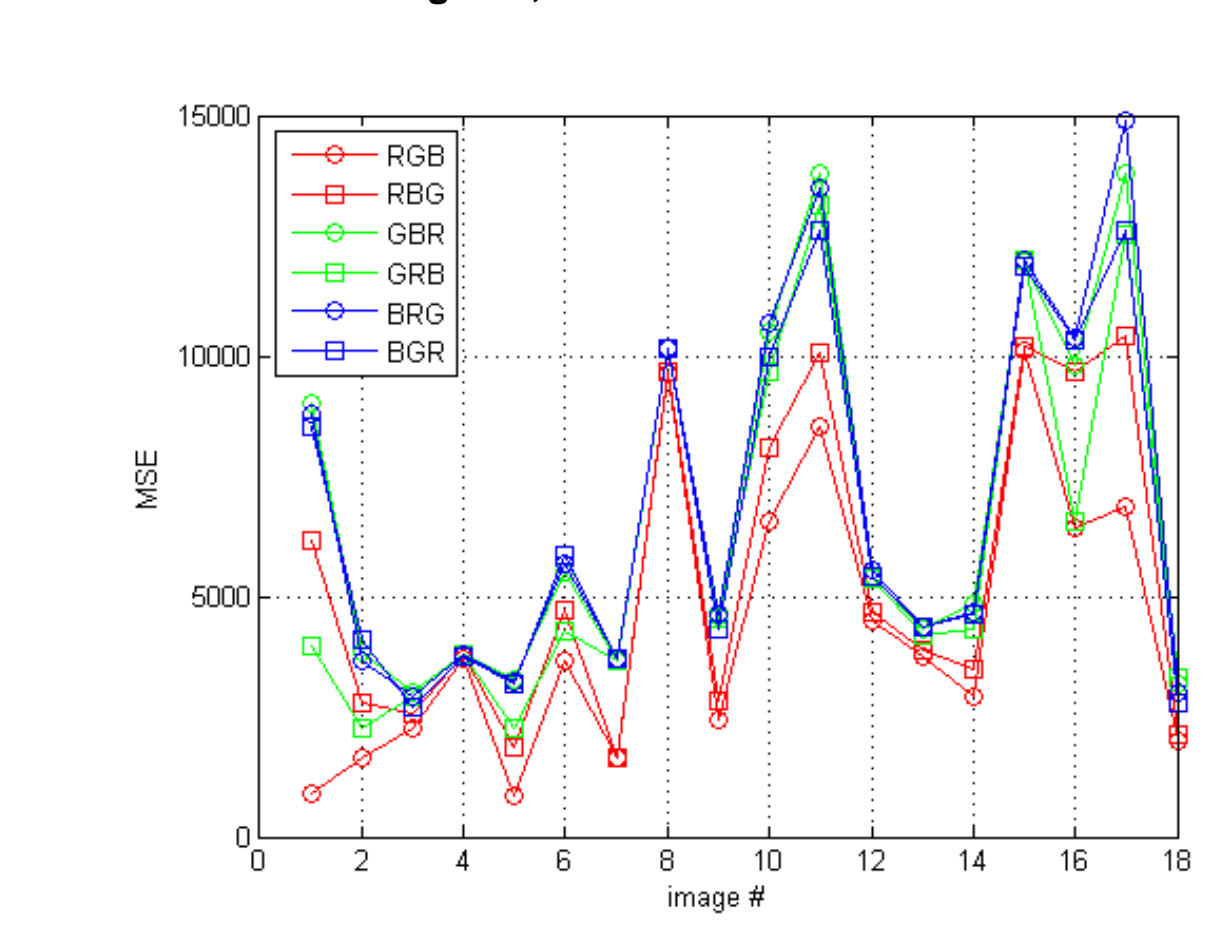

Figure 8. Performance comparison in CMSE: different combination of red, green, and blue combinations 


\section{Conclusions}

This paper presented the effect of color channel replacement with histogram equalization. The proposed system is composed of seven stages, original given image, channel separation: color image to three gray scale image, color channel replacement, HEM on individually separated image, histogram modification in each color channel, reconstruct color images, and image display. The experimental results display the resultant images, and provide PSNR and MSE performance on $18 \mathrm{McM}$ imageset.

\section{Acknowledgements}

This research was supported by Basic Science Research Program through the Natipnal Research Foundation of Korea(NRF) funded by the Ministry of Science, ICT and Future Planning(2013R1A1A1010797)

\section{References}

[1] R. C. Gonzalez and R. E. Woods, "Digital Image Processing", 3rd ed. Upper Saddle River, NJ: Prentice- Hall, (2009).

[2] P. E. Trahanias and A. N. Venetsanopoulos, "Color image enhancement thrøugh 3-D histogram equalization", in Proc. 15th IAPR Int. Conf. Pattern Recognit, vø1. (1992) Aůgus-September, pp. 545-548.

[3] G. Healey, "Segmenting images using normalized color", IEEE Trans. Syst., Man, Cybern., vol. 22, (1992), pp. 64-73.

[4] T. Kim and H. S. Yang, "A Multidimensional histogram equalızation by fitting an isotropic Gaussian mixture to a uniform distribution", in Proc. Int. Conf. Image Process. (2006) October, pp. 2865-2868.

[5] D. Menotti, L. Najman, A. de Araúio and J. Facon, "A fast hue-preserving histogram equalization method for color image enhancement using a Bayesian framewron", in Proc. 14th Int.Workshop Syst., Signal Image Process. (IWSSIP), (2007) June, pp. 414-417

[6] C. L. Huang, T. Y. Cheng and C. C. Chen Color image segmentation using scale space filter and Markov random fields", Pattern Recognit., vol. 25, (1992), pp. 1217-1229.

[7] A. K. Forrest, "Colour histogram equaljsation of multichannel images", in Proc. IEEE Vis. Image Signal Process., vol. 152, N0. 6, (2005) December, pp. 677-686.

[8] I. Pitas and P. Kinikịlis, "Multichannel techniques in color image enhancement and modeling", IEEE Trans. Image Proces vol. 5, no, 1, (1996) January, pp. 168-171.

[9] F. Pitié, A. C. Kokaram (and R. Dahyot, "N-dimensional probability density function transfer and its application to color transfer" in Proc. IEEE Int. Conf. Comput. Vis., vol. 2, (2005) October, pp. 1434-1439.

[10] L. Shafarenko, "Perception-driven automatic segmentation of color images using mathematical morphology", Ph.D. dissertation, Oniv. Surrey, Guildford, U.K., (1996).

[11] P. A. Mlsna and J. I Rodriguez, "A multivariate contrast enhancement technique for multispectral images," IEEE Trans. Geosci. Remote Sens., vol. 33, no. 1, (1995) January, pp. 212-216.

[12] P. A. Mlsna Q Zhang and J. J. Rodriguez, "3-D histogram modification of color images", in Proc. IEEE Int. Conf. Image Process., vol. 3, (1996) September, pp. 1015-1018.

[13] J. Morovic and P. -L. Sun, "Accurate 3-D image colour histogram transformation", Pattern Recognit. Lett., vol. 24, no. 11, (2003) July, pp. 1725-1735.

\section{Author}

\section{Gwanggil Jeon}

He received the $\mathrm{BS}$, MS, and $\mathrm{PhD}$ (summa cum laude) degrees in Department of Electronics and Computer Engineering from Hanyang University, Seoul, Korea, in 2003, 2005, and 2008, respectively.

From 2008 to 2009, he was with the Department of Electronics and Computer Engineering, Hanyang University, from 2009 to 2011, he was with the School of Information Technology and Engineering (SITE), University of Ottawa, as a postdoctoral fellow, and 
from 2011 to 2012, he was with the Graduate School of Science and Technology, Niigata University, as an assistant professor. He is currently an assistant professor with the Department of Embedded Systems Engineering, Incheon National University, Incheon, Korea. His research interests fall under the umbrella of image processing, particularly image compression, motion estimation, demosaicking, and image enhancement as well as computational intelligence such as fuzzy and rough sets theories.

He was the recipient of the IEEE Chester Sall Award in 2007 and the 2008 ETRI Journal Paper Award.

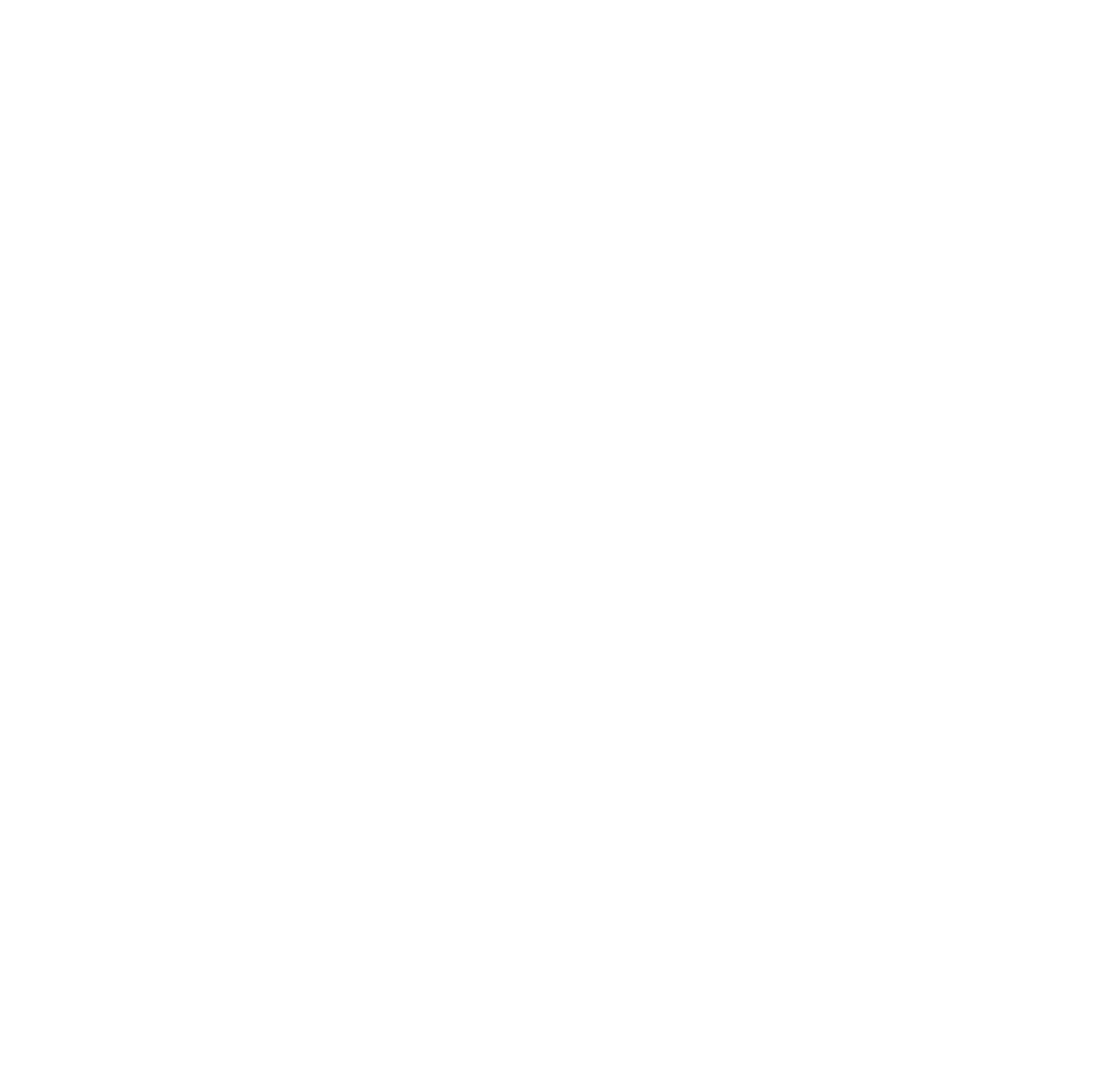

